This item was submitted to Loughborough's Research Repository by the author.

Items in Figshare are protected by copyright, with all rights reserved, unless otherwise indicated.

\title{
Physics of causality and continuum: questioning nature
}

PLEASE CITE THE PUBLISHED VERSION

PUBLISHER

Professional Engineering Publishing / @ IMECHE

VERSION

VoR (Version of Record)

LICENCE

CC BY-NC-ND 4.0

REPOSITORY RECORD

Rahnejat, Homer. 2019. "Physics of Causality and Continuum: Questioning Nature”. figshare. https://hdl.handle.net/2134/4698. 
This item was submitted to Loughborough's Institutional Repository (https://dspace.lboro.ac.uk/) by the author and is made available under the following Creative Commons Licence conditions.

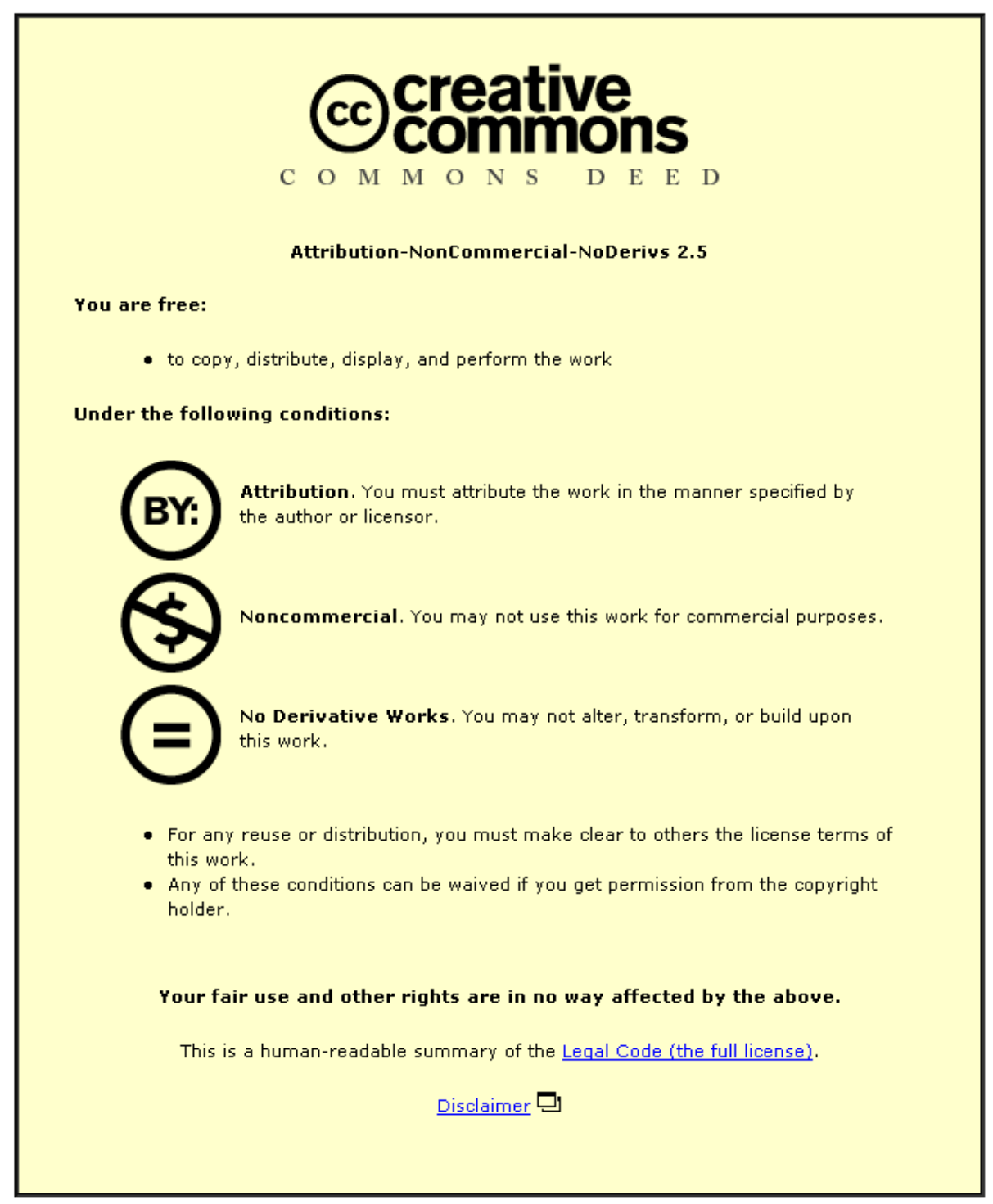

For the full text of this licence, please go to: http://creativecommons.org/licenses/by-nc-nd/2.5/ 


\title{
Physics of causality and continuum: questioning nature
}

\author{
H Rahnejat \\ Wolfson School of Mechanical and Manufacturing Engineering, University of Loughborough, Loughborough, \\ Leicestershire LE11 3TU, UK. email: h.rahnejat@lboro.ac.uk
}

The manuscript was received on 29 May 2008 and was accepted after revision for publication on 3 September 2008.

DOI: 10.1243/14644193JMBD166

\begin{abstract}
This article commemorates three distinct periods in the growing understanding of physics of motion during the Renaissance (referring to advancement of art) period, the age of enlightenment (scientific renaissance: the Newtonian mechanics), and the adoption of field concept, embodied in the theory of relativity. Emphasis is put on Newton's immense and fundamental contributions and those of his immediate and subsequent contemporaries, leading to Lagrangian dynamics, which is the cornerstone of many contributions to this journal. In particular, a generic geometric interaction potential is introduced within a closed field, which conforms to Newton's law of universal gravitation and extends to the scale of microcosm, thus embedding gravity with other forces of nature. The implications of these extensions to the Newtonian potential are discussed.
\end{abstract}

Keywords: atomism, Newtonian mechanics, Lagrangian dynamics, field concept, unified generic interaction potential

\section{INTRODUCTION}

\subsection{Motion as a kinematic concept}

To Aristotle, physics was natural philosophy; study of physical phenomena. This view was later shared by Da Vinci. As the phenomena were considered centred on earth they could be described by hierarchically ordered matter; initially conceived as a Zoroastrian idea, which was adopted by Empedocles and endorsed by Aristotle. This initial atomistic idea of a matterfilled continuum persisted beyond the Copernican heliocentric correction. Galilean principle of relativity [1] and Kepler's explanation of the orbital motions [2] based on the astronomical recordings of Brahe must have given the scholars of the Renaissance quiet satisfaction of a theory of everything in their sights. Nature had yielded its innermost secrets only if the underlying reason could be found in accord with the findings. Descartes' concept of geometrical extension and principle of conservation of motion [3] as the cause underpinned this atomistic view of nature as a supposition: a philosophical view referred to as positivist realism [4]. The affinity of this kinematic explanation to the fundamentals of Euclidian geometry within Cartesian frames of reference was seen as a further proof of its truth [3].

\subsection{Newton and concept of force}

Newton's law of Universal Gravitation, with actionat-a-distance independent of interaction of any intervening matter and their very substance, apart from a quantity defined by him as gravitational mass, came as a shock [5]

$$
F=G \frac{M m_{g}}{r^{2}}
$$

The cause and the continuum were both questioned rocking the very foundations of science as then understood. Newton's implicit appeal to the Galilean principle of relativity and his Euclidean-style proclamation on the axiomatic nature of his laws of motion incensed the Cartesian School. His declarations of action-ata-distance, mass, and force were imponderable declared D'Alembert, adding that mechanics had lost the philosophic basis upon which it had resided prior to Newtonian pragmatic empiricism because it had abandoned the notion of material impulsions necessary for transmission of motion. For Leibniz, the implication of conservation of a kinematic quantity (impulsions) was unthinkable and so was the action-at-a-distance through an aether. In his view, the quantity conserved conditionally was $m v$ (momenta), one favoured by Newton, and unconditionally $m v^{2}$ 
(vis viva: the living force) [6]. Mechanics was neither a question of Cartesian imponderables nor one of pragmatic empiricism: I do not invent hypothesis. D'Alembert's impulsions, he declared were nothing but vis viva. Euler seized on the idea by noting that $F=m a=m \lim _{\Delta t \rightarrow 0}(\Delta v / \Delta t)=m(\mathrm{~d} v / \mathrm{d} t)$ as noted by both Newton and Leibniz and suggested, in a letter to Lagrange, that Newton's force is the same as rate of change of D'Alembert's impulsions. In a response to Lagrange, D'Alembert noted: our friend Euler is a great analyst, but rather a poor philosopher. However, the analyst, not withstanding the Cartesians' objection to the imponderable nature of mass, had unified the views of positivists and the pragmatists alike. D'Alembert restated the Newtonian second axiom by his principle: $F-m(\mathrm{~d} v / \mathrm{d} t)=0$ [7], whereas Euler re-interpreted the Cartesian rigidly transmitted impulsions as constraints [8]. With Coriolis definition of kinetic energy as $(1 / 2) m v^{2}$ all the pieces of jigsaw were available for Lagrange to recreate the lost continuum of D'Alembert with mathematical exposition of Newton's second axiom for particles and bodies alike [9], but with the underlying cause for universal gravitation and mass remaining illusive at the time. Thus, the atomistic view of nature survived the philosophical objections of the period that was regarded as scientific Renaissance or the age of enlightenment. Was this the theory of everything?

It was clearly a better theory of motion than the previous one, which was merely based on kinematics quantities - as it addressed the underlying cause - mainly the concept of force; a theory generally termed as Newtonian mechanics. However, one should not under-estimate the contribution of Galileo as well as Newton's contemporaries and others as highlighted above, as any theory should have a strong philosophical basis. The philosophical basis for the classical theory is atomistic, which in time became its Achilles' heel. For most of the seventeenth, eighteenth, and nineteenth centuries, however, it transformed the human perception of nature, not only those engaged in science but of everyone. An age of enlightenment was declared by the influential scholars of the time from all walks of life such as Voltaire who, referring to Newton, declared: we are all his disciples, and Delille: Glory to God who created the worlds and Newton and Butterfield on the effect of Newton's works: ... changed the character of men ... and the very texture of human life itself.

The atomistic view of the universe was accepted in a continuum devoid of matter for the lynchpin of Newtonian mechanics; the law of universal gravitation to be upheld. The Cartesian matter-filled universe was replaced by the concept of aether, which was generally accepted out of necessity and not of any conviction. On the contrary, what constitutes a continuum of nothing? How may the principle of causality be upheld for an action-at-a-distance through such vacuo? The concerns gathered pace with the discoveries of Coulomb [10] and Maxwell [11] in the nineteenth century. Significant forces other than gravity were discovered, and while electrostatics was formulated in a similar inverse distance squared law as that for gravity, the constant of proportionality $K$ was a function of the medium in which the charges resided unlike $G$ the universal gravitational constant, which is independent of the fabric of space or material of bodies. Note [10]

$$
F=\frac{K q_{2}}{r^{2}}
$$

where $K=q_{1} / 4 \pi e_{0} e_{\mathrm{r}}$.

\subsection{Emerging problems with Newtonian mechanics}

With the discovery of electromagnetism - mobile charges and wave motions within a field (first discovered by Faraday) - the age old concerns with regard to the concepts of infinity and infinitesimal returned to the fore. With the realization of elementary particles and wave motion taking place at or near the speed of light other fundamental concerns emerged. The predominant worry was that the second Newtonian axiom returned an infinite velocity for a massive body subjected to an inappreciable force, providing such a force was applied for a long enough duration

$$
v=\frac{F}{m_{I}} t_{\mid v=0, t-0} t \rightarrow \infty \mid v \rightarrow \infty
$$

where Newton referred to the mass of the object in his second axiom as inertial, and as distinct from that in equation (1). It is variously suggested that this anomaly in dual description of mass remained until its resolution in the early twentieth century. However, Newton asserted [5]: Not that I affirm gravity to be essential to bodies: by their vis insita I mean nothing but their vis inertiae. This is immutable. In Principia, Newton refers to the equality of these masses having carried out some computations, without going into any mathematical discourse. Newton stated: Therefore if, at equal distant from the Sun, the accelerative gravity of any satellite towards the Sun were greater or less than the accelerative gravity of Jupiter towards the Sun but by one 1/1000 part of the whole gravity, the distance of the centre of the satellite's orbit from the Sun would be greater or less than the distance of Jupiter from the Sun by one 1/2000 part of the whole distance; that is, by a fifth part of the distance of the utmost satellite from the Jupiter; an eccentricity of the orbit which would be very sensible. In fact, Chandrasekhar [12] surmises Newton's 'computations', arriving at a solution to show Newton's doubtless awareness of the 
same. Newton's assertions can be justified by an even simpler attempt. It is clear by virtue of the law of universal gravitation that the force acting on a body on the surface of the earth (weight) is: $F=\left(G M / r^{2}\right) m_{g}$. This force must comply with Newton's second axiom, thus: $F=m_{I} g$ ( $m_{L}$ being its vis inertiae $)$. One can conclude that: $g=\left(G M / r^{2}\right)\left(m_{g} / m_{I}\right)=D \bar{m}$, where $D$ is a constant for $r$, radius of the earth; $G$, universal gravitational constant; and $M$ mass of the earth being all constants. Now, various bodies having different ratios $\bar{m}$ imply that they would fall to the ground at different rates $g$ (which has never been sensed) - an Aristotelian view which was rightly refuted by Galileo in 1589 .

\subsection{Atomism versus field concept}

Somehow the duality of definition of mass, in the absence of a mathematical disposition, seems to have escaped the critical assessment of the scientific renaissance. For one reason, D'Alembert viewed mass as imponderable not an intrinsic property of matter. This view was clearly shared by Mach [13] much later. He noted that if two different forces should act on different bodies, but this time in a manner to accelerate the bodies at the same rate, then the second axiom can be restated as: $\bar{m}=m_{1} / m_{2}=C^{\prime}\left|F_{1} / F_{2}\right|=C^{\prime}|\bar{F}|$, with $\left|F_{1} / F_{2}\right|=|\bar{F}|=\left|a_{1} / a_{2}\right|$. Thus, to Mach mass was not an intrinsic property, but a linkage between a body and all other bodies that interact with it and exert force upon it. This definition was contrary to the prevailing atomistic view at the time. It may be regarded as a closed system or a field view.

Whether or not the equality of inertial and gravitational mass was fully appreciated in the seventeenth and eighteenth centuries, any ambiguity was resolved with Einstein's Equivalence principle [14]. The essence of this principle is the equivalence of two systems: one fixed in a gravitational field and the other falling uniformly with an equivalent acceleration. Einstein then concluded: This assumption of exact equivalence makes it impossible for us to speak of the absolute acceleration of a system of reference, just as the usual theory of relativity forbids us to talk of the absolute velocity of a system; and makes the equal falling of all bodies in a gravitational field seem a matter of course.

\subsection{Relative bounded motion: special theory of relativity}

Clearly, motion of observed matter must remain within the limit of observation itself. This means that speed of moving matter cannot exceed that of light (the medium of observation). Therefore, in the space-time continuum events take place within the light-cone with the speed of light being the same and a constant for all observers in relative motion. Einstein declared this as a law of physics as well as the Galilean principle of relativity [15]. Laws of physics should be underpinned with a philosophical as well as mathematical basis as is already clear from the foregoing. The theory to uphold the stated laws is the special theory of relativity, which needed to describe the relative nature of space, time, motion of observers, mass, and momenta. To achieve this, the Galilean transformation gave way to the Lorentzian transformation and Euclidean geometry to one of the hyperbolics [16]. Einstein also addressed the issue of inertia of bodies by noting that it would depend on their velocity within the light-cone [15, 17]. Referring to the special theory of relativity he states [17]: If the theory corresponds to the facts, radiation conveys inertia between the emitting and absorbing bodies. This leads to his famous equation: $E=m c^{2}$. Description and implications of special theory of relativity have recently appeared in this journal's commemorative issue $[\mathbf{1 8}, \mathbf{1 9}]$. The key point emerging from the special theory was its applicability to inertial frames of reference only (i.e to observers in uniform relative state of motion) because it is a theory based on kinematics.

\subsection{Field theory for gravitation and explanation for action at a distance}

To account for non-inertial frames of reference Einstein had to reconcile the theory with accelerated motions. Note that in Fig. 1, the asymptotes represent the limiting speed (that of light represented by the slope of unity), showing a $(1+1)$ space-time of an inertial observer $(t, z)$. Then, world-curve of motion of all matter other than light is bounded within the cone. These typical hyperbolic worldcurves are of the form $s(\tau)=(t(\tau), z(\tau))$, and with the proper velocity of a material point along the curve

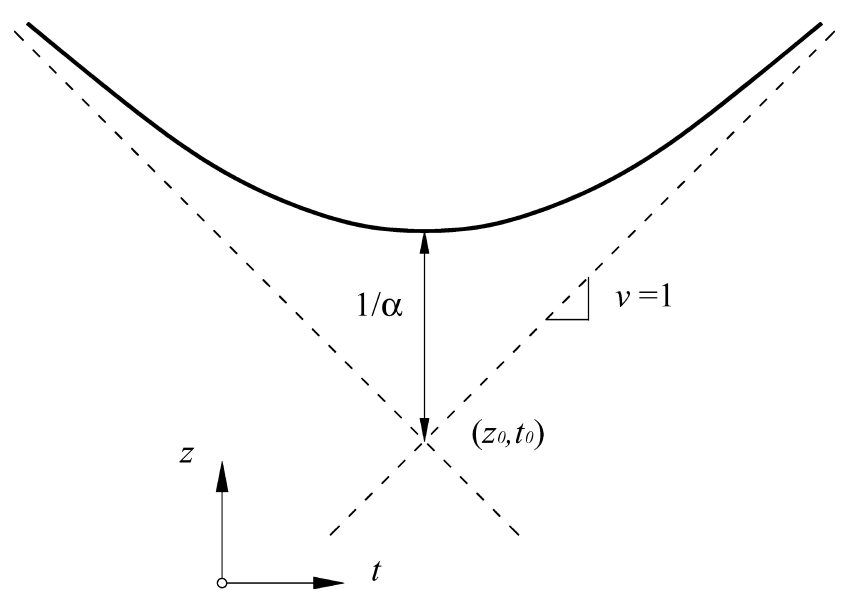

Fig. 1 Accelerated motions curve away from slope of unity 
given as: $u(\tau)=(\mathrm{d} t / \mathrm{d} \tau, \mathrm{d} z / \mathrm{d} \tau)=\mathrm{d} t / \mathrm{d} \tau(1, v / c)$, with the velocity limitation of: $\|u(\tau)\|^{2} \equiv 1$, thus $\mathrm{d} t / \mathrm{d} \tau=1$ / $\sqrt{1-v^{2}}, v=v / c$, which is termed the local time dilation factor. The world-curve deviating from linearity represents accelerated motion of a material point. Assuming constant acceleration, with a magnitude $a=\|a(\tau)\|$, thus

$$
\frac{\mathrm{d}^{2} z}{\mathrm{~d} \tau^{2}}-\frac{\mathrm{d}^{2} t}{\mathrm{~d} \tau^{2}}=a^{2}
$$

Proper parameterization of the world-curve in terms of proper time $\tau$ constitutes a function $f(\tau)$, thus $\mathrm{d} t / \mathrm{d} \tau=\cosh f(\tau)$ and $\mathrm{d} z / \mathrm{d} \tau=\sinh f(\tau)$.

Differentiating $\mathrm{d}^{2} t / \mathrm{d} \tau^{2}=f^{\prime}(\tau) \sinh f(\tau), \mathrm{d}^{2} z / \mathrm{d} \tau^{2}=$ $f^{\prime}(\tau) \cosh f(\tau)$, and thus implying that $f^{\prime}(\tau)= \pm a$ and $f(\tau)= \pm a\left(\tau+\tau_{1}\right)$, with $\tau_{1}$ being a constant of integration. Inserting this into equation (4) and integrating twice one can find $t(a)$ and $z(a)$, which leads to $z=z_{0} \pm(1 / a) \sqrt{1+a^{2}\left(t-t_{0}\right)^{2}}$, and as $t \rightarrow \infty$ unlike equation (3), $\|\mathrm{d} z / \mathrm{d} \tau\|=v \rightarrow \pm 1$ or $v \rightarrow \pm c$. This analysis shows that accelerated motions take place in curved space-time and within the limit set by the light cone. Only a very small region of space-time is considered here, where a constant acceleration may be assumed. However, the same can also be proven for other accelerated frames. Thus, accelerated motions are linked to curvatures of space-time. In the example described here, motion of the body is described in the coordinate system $(\tau, \zeta)$ and observed from the inertial frame of reference $(t, z)$. The findings are true for any arbitrary frame leaving Einstein to make the profound statement [14]: It will be seen from these reflections that in pursuing the general theory of relativity we shall be led to a theory of gravitation since we will be able to 'produce' a gravitational field merely by changing the system of coordinates - for example, from $(\tau, \zeta)$ to $(t, z)$, noting that by virtue of the equivalence principle inertial acceleration is the same as an induced gravitational field.

It is clear that although acceleration is linked to curvature, the underlying cause of curved spacetime must be explained by a theory for gravity not one based on atomistic explanation of action-ata-distance, but by a field. Einstein's hitherto atomistic view in 1905 changed when he was particularly struck by Mach's closed system philosophy [20]: From Newton's time on, the theory of action-at-a-distance was constantly found artificial. ...The strange part played by space (or the inertial system) within the mechanical foundation was also clearly recognized, and criticized with especial clarity by Ernst Mach. This statement reflects, retrospectively, Einstein's state of mind in the period 1908-1911 in a search for a field concept for gravity which would be befitting of Faraday's discovery of electromagnetic fields [20]: For us, who took in Faraday's ideas so to speak with our mother's milk, it is hard to appreciate their greatness and audacity. To achieve his wish, a new formulation was needed for the law of inertia, which in the absence of a gravitational field would uphold the Galilean principle of inertia, which states that with no net force the shortest line in space-time, termed as the extremal line, would be a straight one. The length of such a line would be a metric, $\mathrm{d} s$. Now if a mere change in coordinates produces a gravitational field then a non-linear transformation $\mathrm{d} s^{2}$ should exist which would remain a homogeneous function of the differentials of the coordinates, which is in fact a Riemannian metric [21]: $\mathrm{d} s^{2}=\sum_{i j} \boldsymbol{g}_{i j} \mathrm{~d} \mathbf{x}_{i} \mathrm{~d} \mathbf{x}_{j}$, where the summation is extended to all indices 1,1 to 4,4 for $(1+3)$ space-time. The metrical coefficients $\boldsymbol{g}_{i j}$ describe the gravitational field with reference to the selected coordinates. A field theory was thus established for gravitational action in terms of curvatures of space compelling motion of material points upon them without resorting to atomism of action-at-adistance. The field concept underpins the principle of causality and retains the Newtonian concept of continuum, but keeps the concept of mass and fails to explain the observed quantized nature of energy in scale of minutia. Thus, the atomistic view lives on within the rival theory of quantum mechanics eventhough it has failed to provide an explanation beyond the very confines of microcosm. Discussion of quantum mechanics is beyond the scope of this article, and the theory of relativity is not regarded as that of everything because of it. It is unlikely that either theory would emerge as unfounded. On the contrary, the resolution of concept of mass would pave the way for the rival philosophical views; atomism or field concept, to gain in ascendancy. For the atomistic view to prevail, the existence of sub-atomic particles/strings have to be verified beyond mere conjecture in the same manner that bending of light rays provided proof of implications of general theory of relativity. In particular, a proof for the conjectured mass-giving particle invariably referred to as graviton would be essential. For the field concept, mass should be proved to be a property of a space-matter closed field or an imponderable necessity.

Although the field view of accelerated motion changed the Newtonian mechanical perspective, much of Newton's mechanics still form the basis of applied physics including most of dynamics. On the two hundredth anniversary of his death Einstein wrote [22]: he was marvelously inventive as regards detailed mathematical and physical methods of proof. For all these reasons he deserves our deepest reverence. 


\section{MATHEMATICAL EXPOSITIONS}

Lagrange's proof of Newton's second axiom, commencing from kinetic and potential energies of matter, encapsulates the efforts of all his contemporaries and arguably captures the scientific advances of the age of enlightenment. The derivation in [9] can be made as an allusion to relativity and within a field concept. It can also be extended as a deviation from a unified frame (that of observation itself). This forms the basis of the mathematical exposition presented here.

\subsection{Lagrange on Newton's second axiom: an allusion to relativity}

Generally, the instantaneous motion of any particle or body moving along a curvilinear path can be parameterized on a localized surface $\mathrm{x}$, termed as the tangent plane to a small region of three-dimensional space. Then, the regional space-time can be given as $\mathrm{x}=\mathrm{x}\left(q^{i}\right)$. The velocity of the particle is then obtained as a pair of tangents to this parameterized space as [19]

$$
\boldsymbol{v}^{i}=\frac{\partial \mathbf{x}}{\partial t}=\frac{\partial \mathbf{x}}{\partial q^{i}} \frac{\mathrm{d} q^{i}}{\mathrm{~d} t}=\mathbf{x}_{i} \dot{\boldsymbol{q}}^{i}=c \mathbf{x}_{i} \frac{\partial q^{i}}{\partial \zeta}
$$

and similarly

$$
\boldsymbol{v}^{j}=\mathbf{x}_{j} \dot{q}^{j}=c \mathbf{x}_{j} \frac{\partial q^{j}}{\partial \zeta}
$$

Note that the space-based definition of velocity is used with respect to the slope of unity described in reference [19] as $\dot{q}^{i}=c\left(\partial q^{i} / \partial \zeta\right)$.

Now, the proper velocities of a material point needs to be determined with respect to its proper time, $\tau$. Admitting only small changes within the light cone, those in proper time are given in terms of a mix of infinitesimal changes with respect to the space-time coordinates $\left(\xi^{0} \equiv t, \zeta \equiv \xi^{i}, i=1 \rightarrow 3\right)$ as $c^{2} \mathrm{~d} \xi^{0^{2}}-\mathrm{d} \zeta^{2}=\mathrm{d} \tau^{2}$. Thus, the proper velocities are obtained as [19]

$$
\boldsymbol{u}^{i}=\frac{\partial \mathbf{x}}{\partial \tau}=\frac{\partial \mathbf{x}}{\partial t} \frac{\partial t}{\partial \tau}=\frac{1}{I^{1}} \boldsymbol{v}^{i}=\frac{1}{I^{1}} c \mathbf{x}_{i} \frac{\partial q^{i}}{\partial \zeta}
$$

and similarly

$$
\boldsymbol{u}^{j}=\frac{1}{I^{1}} c \mathbf{x}_{j} \frac{\partial q^{j}}{\partial \zeta}
$$

Since $\mathrm{d} t / \mathrm{d} \tau=1 / \sqrt{1-v^{2}}, v v / c, v=|\boldsymbol{v}|$ and deviation from the slope of unity is obtained as $I^{1}=\sqrt{1-v^{2}}$
Now, acceleration is obtained as

$$
\begin{aligned}
\boldsymbol{a}^{i} & =\left(\frac{1}{I^{1}}\right)^{2} \frac{\partial^{2} c^{2} \mathbf{x}^{i}}{\partial \zeta^{2}}=\left(\frac{1}{I^{1}}\right)^{2} \frac{\partial}{\partial \zeta}\left(c^{2} \frac{\partial \mathbf{x}^{i}}{\partial \zeta}\right) \\
& =\left(\frac{1}{I^{1}}\right)^{2} \frac{\partial}{\partial \zeta} \underbrace{\{c^{2} \underbrace{\left(\mathbf{x}_{i} \frac{\partial q^{i}}{\partial \zeta}+\mathbf{x}_{j} \frac{\partial q^{j}}{\partial \zeta}\right)}_{\nabla s}\}}_{\varphi}=\left(\frac{1}{I^{1}}\right)^{2} \nabla \varphi
\end{aligned}
$$

where $\nabla s$ is the curvature of the tangent plane with the basis $\mathbf{x}_{i}, \mathbf{x}_{j}$.

Using Bernoulli's principle of virtual work [23] for static equilibrium (initially expounded in 1717) $\delta \mathbf{W}=$ $\sum_{i} \mathbf{F}_{i} \cdot \delta \mathbf{r}_{i}$, where the summation over the repeated index $i$ can be removed according to Einstein's tensor notation, thus $\delta \mathbf{W}=\mathbf{F}_{i} \cdot \delta \mathbf{x}_{i}=\mathbf{F}_{i}\left(\partial \mathbf{x}_{i}\right) /\left(\partial q^{k}\right) \delta q_{k}=$ $\mathbf{F}_{i} \mathbf{x}_{, k}^{i} \delta q^{k}$.

The D'Alembert principle extends the above case to one of dynamics [7]

$$
\left(\mathbf{F}_{i}-\mu_{k} \frac{\partial^{2} \mathbf{x}_{\mathrm{i}}}{\partial t^{2}}\right) \delta \mathbf{x}_{\mathrm{i}}=0
$$

where in terms of coordinates $q^{i}, \dot{\mathbf{x}}$ values for these coordinates are given by equations (5) and (6). The second term in the equation expands as

$$
\begin{aligned}
& \mu_{k} \frac{\partial^{2} \mathbf{x}_{i}}{\partial t^{2}} \delta \mathbf{x}_{i}=\mu_{k} \frac{\partial^{2} \mathbf{x}_{i}}{\partial t^{2}} \mathbf{x}_{, k}^{i} \delta q^{k} \\
& \quad=\left\{\left[\left(\frac{\mathrm{d}}{\mathrm{d} t}\right)\left(\frac{\partial}{\partial \dot{\boldsymbol{q}}^{k}}\right)-\left(\frac{\partial}{\partial q^{k}}\right)\right]\left(\frac{1}{2} \mu_{k} v^{i} v^{j}\right)\right\} \delta q^{k}
\end{aligned}
$$

and as it can be seen one arrives at the Lagrange's equation, if replaced in equation (10), observing that $\mathbf{F}_{i} \mathbf{x}_{, k}^{i}=\mathbf{F}_{k}$ with $\mathbf{F}_{k}=-\left(\partial U / \partial q^{k}\right)$ as noted by Euler $[\mathbf{8}]$. Now noting that the first pair of differentials in the curly bracket delivers $m_{k} \boldsymbol{a}^{k}$ and that kinetic energy is independent of choice of $q^{k}$, the philosophic-mathematical basis for Newton's second axiom is thus established. However, the problem with $\boldsymbol{v}^{i} \rightarrow \infty$ exists until the proper time $\tau$ is taken into account.

Kinetic energy is obtained as

$$
\begin{aligned}
T & =\frac{1}{2} \mu \boldsymbol{u}^{i} \cdot \boldsymbol{u}^{j}=\frac{1}{2} \mu \mathbf{x}_{i} \cdot \mathbf{x}_{j}\left(\frac{1}{I^{1}}\right)^{3} c^{2} \frac{\partial q^{i}}{\partial \zeta} \frac{\partial q^{j}}{\partial \zeta} \\
& =\frac{1}{2}\left(\frac{1}{I^{1}}\right)^{3} \mu c^{2} \mathbf{g}_{i j} \frac{\partial q^{i}}{\partial \zeta} \frac{\partial q^{j}}{\partial \zeta}
\end{aligned}
$$

where $\mathbf{g}_{i j}=\mathbf{x}_{i} \cdot \mathbf{x}_{j}$ is the first fundamental form (metric tensor for the parameterization $\mathbf{x}$ ). 
Now refer back to the terms in the Lagrange's equation [9] to note that

$$
\frac{\mathrm{d}}{\mathrm{d} \tau}\left(\frac{\partial T}{\partial \dot{q}^{k}}\right)=\left(\frac{1}{I^{1}}\right)^{3} \mu c^{2}\left(\mathbf{g}_{k j} \frac{\partial^{2} q^{k}}{\partial \zeta^{2}}+\frac{\partial q^{k}}{\partial \zeta} \frac{\partial q^{j}}{\partial \zeta} \frac{\mathrm{d} \mathbf{g}_{k j}}{\mathrm{~d} \zeta}\right)
$$

and with a change of the base to $\zeta$

$$
\frac{\partial T}{\partial q^{k}}=\frac{1}{2}\left(\frac{1}{I^{1}}\right)^{3} \mu c^{2} \frac{\partial \mathbf{g}_{i j}}{\partial \zeta} \frac{\partial q^{i}}{\partial \zeta} \frac{\partial q^{j}}{\partial \zeta}
$$

Now, replacing in Lagrange's equation and denoting partial differentials with respect to the spatial coordinates $\zeta$

$$
\begin{aligned}
& \frac{\mathrm{d}}{\mathrm{d} t}\left(\frac{\partial T}{\partial \dot{q}^{k}}\right)-\frac{\partial T}{\partial q^{k}}-\mathbf{F}_{k} \\
& \quad=\left(\frac{1}{I^{1}}\right)^{3} \mu c^{2}\left\{\mathbf{g}_{k j} q^{\prime \prime k}+q^{\prime k} q^{\prime j} \mathbf{g}_{k j}^{\prime}-\frac{1}{2} \mathbf{g}_{i j}^{\prime} q^{\prime i} q^{\prime j}\right\}-\mathbf{F}_{k} \\
& \quad=\left(\frac{1}{I^{1}}\right)^{3} \mu c^{2}\left\{\mathbf{g}_{k j} q^{\prime \prime k}+\frac{1}{2}\left(\mathbf{g}_{k j}^{\prime}+\mathbf{g}_{k i}^{\prime}-\mathbf{g}_{i j}^{\prime}\right) q^{\prime i} q^{\prime j}\right\}-\mathbf{F}_{k} \\
& =\left(\frac{1}{I^{1}}\right)^{3} \mu \underbrace{\mathbf{g}_{k n} \underbrace{\left(q^{\prime \prime n}+\Gamma_{i j}^{n} q^{\prime i} q^{\prime j}\right) c^{2}}_{\mathbf{a}^{n}}-\mathbf{F}_{k}}_{\nabla h} \\
& \quad=m \boldsymbol{a}^{k}-\mathbf{F}_{k}=0
\end{aligned}
$$

where $\Gamma_{i j, n}=1 / 2\left(\mathbf{g}_{k j}^{\prime}+\mathbf{g}_{k i}^{\prime}-\mathbf{g}_{i j}^{\prime}\right)$ is the Christoffel tensor of the first kind modified to refer back to the coordinate set $\left(\xi^{0} \equiv t, \zeta \equiv \xi^{i}, i=1 \rightarrow 3\right)$ for absolute motion, and $\Gamma_{i j}^{n}=\mathbf{g}^{k n} \Gamma_{i j, n}$ the Christoffel symbol of the second kind.

\subsection{A generic interaction potential}

It is now appropriate to assume a potential of general form: $\varphi=-\sum_{i=1}^{n} C \varepsilon^{i-1} / r^{i}=-\left(r^{n}-\varepsilon^{n}\right) / r^{n}(r-\varepsilon)$, where $\varepsilon$ is the size of the body and $r$ a region of space around it. For the Newtonian gravitational potential: $C=G M$ where $G$ is the universal gravitational constant and $M$ is the mass of a source. This form of potential makes the constant $C$ of the correct units for the inverse ascending powers of $n$.

Acceleration is expressed as $a=-\partial \varphi / \partial r$ and using the same generic form of potential

$$
\begin{aligned}
a & =-C \frac{\partial}{\partial r}\left(\sum \frac{\varepsilon^{n-1}}{r^{n}}\right)=-C \frac{\partial}{\partial r}\left(\frac{\left(r^{n}-\varepsilon^{n}\right)}{r^{n}(r-\varepsilon)}\right) \\
& =-C \sum_{1}^{\infty} \frac{n \varepsilon^{n-1}}{r^{n+1}}
\end{aligned}
$$

when $n=1, a=C / r^{2}$, which is the gravitational acceleration $\left(a=g=G M / r^{2}\right)$. Note that gravitational acceleration is independent of the size of the body $\varepsilon$. However, this is not the case for the higher values of $n$. In a system of size $\ell, r=\ell-\varepsilon$. Replacing for $r$ in equation (15)

$$
\begin{aligned}
a & =-\frac{C}{\varepsilon^{2}} \sum_{1}^{\infty} \frac{n}{[(\ell / \varepsilon)-1]^{n+1}} \\
& =-\frac{C}{\varepsilon^{2}} \sum_{1}^{\infty} \frac{n}{[1-\varepsilon / \ell]^{n+1}}\left(\frac{\varepsilon}{\ell}\right)^{n+1}
\end{aligned}
$$

For $\ell / \varepsilon \gg 1$, this expression simplifies to

$$
a=-\frac{C}{\ell^{2}} \sum_{1}^{\infty} n\left(\frac{\varepsilon}{\ell}\right)^{n-1}
$$

Note that the interactions are dominated by its first term (i.e. the gravitational acceleration). When $p=$ $\ell / \varepsilon \rightarrow 1$, the limit of convergence for the series in equation (16) can be obtained by the D'Alembert test ratio as

$$
\begin{aligned}
\operatorname{Lt}_{n \rightarrow \infty} \frac{u_{n}}{u_{n-1}} & =\operatorname{Lt}_{n \rightarrow \infty} \frac{n(p-1)^{n}}{(n-1)(p-1)^{n+1}} \\
& =\operatorname{Lt}_{n \rightarrow \infty} \frac{n}{(n-1)(p-1)} \Rightarrow p>2 \Rightarrow \frac{\ell}{\varepsilon}>2
\end{aligned}
$$

Figure 2 shows the variation of acceleration ratio $\bar{a}=a \varepsilon^{2} / C$ with $\varepsilon / \ell=1 / p$ for the ascending powers of $n$. The variations indicated by any set of combined indices extending to a particular value of $n$ correspond to the attractive interactions given by equation (16). Those indicated by $n^{\prime}$ also include repulsive action at close range, which is discussed later. As the ratio $1 / p$ increases, the contributions due to higher values of $n$ dominate. Larger values of the ratio correspond to interactions at closer range, typical of small material

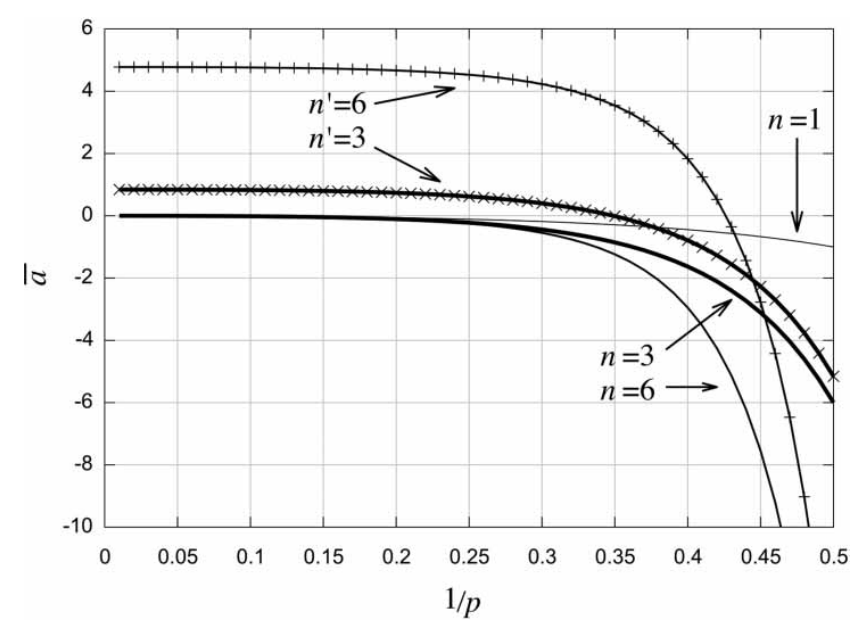

Fig. 2 Dimensionless acceleration due to various combinatorial interaction potentials 
points (particles or molecules). At longer range, corresponding to larger bodies (smaller values of the ratio), the contributions of higher potential powers merge into gravitational action. In such cases, as shown above, the interactions are independent of the size of the body. Newton referred to this attribute of gravity as: a non-discriminatory force of Nature. Smaller bodies act with the dominance of greater values of $n$. In a system of size $\ell$, a body $\varepsilon$ is subjected to interactions with all the other surrounding matter with different powers of $n$. Therefore, its acceleration is the measure of all such interactions in an assumed closed system (the basis of Mach's principle as declared by Einstein). The dominant interaction for such a body will be according to its size and its closeness to its immediate neighbours. These characteristics are captured in Fig. 2 and are continuous in form, upholding Newton's continuum unconditionally and providing unification of gravity with other forces of Nature. When the ratio $\varepsilon / \ell \rightarrow 1 / 2$, the work required to overcome the mutual attraction would tend to infinity with $n \rightarrow \infty$ (equation (18)). This condition can presumably occur for very small elementary particles, e.g. corresponding to the strong force at the atomic nuclei. It is important to note that so far the expounded generic potential does not include repulsion at close range.

Equation (18) has the limiting D'Alembert ratio of unity for smaller values of $\ell / \varepsilon$ (convergence of finite series). Thus, the D'Alembert test ratio yields the limiting relationship $p=\ell / \varepsilon=2 n-1 / n-1$. For $n=1, \ell / \varepsilon \rightarrow \infty$, which means that it can assume any value (it is non-discriminatory according to Newton).

For $n=2,3,4,5, \ldots, \rightarrow \infty, \ell / \varepsilon=3,5 / 2,7 / 3,9 / 4, \ldots$, $\rightarrow 2$. It is, therefore, clear that interaction energies with higher inverse powers of distance (higher values of $n$ ) act in a progressively shorter range and in an attractive manner up to the stated limits. Following that, their contributions to the overall interactions become repulsive. Therefore, equation (16) can be modified to

$$
\begin{aligned}
a=-\frac{C}{\varepsilon^{2}}\{ & \frac{1}{[\ell / \varepsilon-1]^{2}} \\
& \left.+\sum_{2}^{\infty} n\left[\frac{1}{(\ell / \varepsilon-1)^{n+1}}-\frac{1}{(n /(n-1))^{n+1}}\right]\right\}
\end{aligned}
$$

Figure 2 also includes the acceleration ratio $\bar{a}$ for this overall potential shown by combination interactions up to the index $n^{\prime}$. It is clear that for gravitational acceleration the overall potential is the same as the gravitation (i.e. $n^{\prime}=1$ is the same as $n=1$ ). This is not true for any other combination $n$ (cases for $n^{\prime}=3$ and $n^{\prime}=6$ are shown in Fig. 2). For $n^{\prime}=6$ the interactions are at closer range and the repulsion limit is at a higher value of $\varepsilon / \ell$. Therefore, when repulsive potential occurs at higher values of $n$, the lower potential indices continue to cause attraction (for $n=1$ this is always the case). The net interactions, therefore, depend on the body size $\varepsilon$ and its interactions with its neighbours in an assumed system of size $\ell$. If an upper limit of $n^{\prime}=6$ is assumed, then it is clear that repulsion occurs due to $n=6,5,4$, whereas interactions still remain for $n=1,2,3$ down to $\varepsilon / \ell=0.4$. Residual attraction due to gravity $(n=1,2)$ means that an equilibrium is reached at a lower value of the ratio (Fig. 2). Since $\bar{a} \propto \varepsilon^{2}$ at close range (for small bodies) $\Delta a \propto$ $1 / \varepsilon^{2} \Delta \bar{a}$ and the interaction ratio between $n^{\prime}=6$ and gravity $(n=1)$ for a molecule of size $\approx 1 \mathrm{~nm}$ would be: $\approx 4 C \times 10^{18}$. This serves the purpose of demonstrating the weak nature of gravity at close range.

\subsection{Interactions in Mach's closed system interpretation}

Now equating (9) and (19) with the former arising from a closed field interpretation (in accord with Mach's principle [19]) and the latter being that due to the generic interaction potential, yields

$$
\begin{aligned}
\frac{c^{2} \varepsilon^{2} \nabla s}{C}=- & \left\{\frac{1}{[\ell / \varepsilon-1]^{2}}+\sum_{2}^{\infty} n\right. \\
& \left.\times\left[\frac{1}{(\ell / \varepsilon-1)^{n+1}}-\frac{1}{(n /(n-1))^{n+1}}\right]\right\}
\end{aligned}
$$

where $\left(1 / I^{1}\right)^{2}=\left(1-v^{2}\right)^{-1}=1+v^{2}+v^{4}+v^{6}+\cdots \approx 1$ for $v \ll 1$.

$$
\text { For } \frac{\ell}{\varepsilon} \gg 1 \text { and } n=1: \frac{\ell^{2} c^{2} \nabla s}{C}=-1
$$

This is a non-dimensional acceleration ratio within a closed field: due to the electromagnetic potential $\ell c^{2} \nabla s$ over an attractive gravitational potential $C / \ell$ in the same system of size $\ell[\mathbf{1 9}]$. The former encourages dispersion whereas the latter promotes cohesion. In Mach's interpretation [13] (section 1.4) this ratio corresponds to two forces: one tending to gravitate all matter within the system $\ell$ and the other attempting to disperse them. It can be seen that the aforementioned forces equilibrate within a gravitational-type field for material points with $v \ll 1$ and $\ell / \varepsilon \gg 1$. A good example of this is the balance of gravitation and electromagnetic repulsion at the surface of stars mitigating their implosion or explosion (for $n$ and $n^{\prime}$ being the same for the value of unity, also confirmed by equation (21)). For other combinations of values of $n$

$$
\left(\frac{1}{I^{1}}\right)^{2} \frac{c^{2} \varepsilon^{2} \nabla s}{C}=\bar{a}
$$

This ratio follows the characteristics in Fig. 2 for $v \ll 1$. Now the local curvature was obtained in reference [19] 
as

$$
I^{2}=\frac{\partial^{2} s}{\partial \zeta^{2}}=I^{1^{2}} \frac{a}{c^{2}}=\frac{\nabla \varphi}{\varphi}
$$

Note that $I^{2}$ is the curvature deviation from the slope of unity (light cone, Fig. 1).

Using equations (22) and (23) and replacing for $\bar{a}=$ $a \varepsilon^{2} / C$, it follows

$$
\nabla s=I^{2}=I^{1^{2}} \frac{a}{c^{2}}=\frac{\nabla \varphi}{\varphi}
$$

The ratio $\nabla \varphi / \varphi$ is the curvature of the local potential field due to material points in a system of size $\ell$ with respect to the slope of unity, where the curvature $I^{2}=0$ (light-like behaviour). The implication of this is shown in Fig. 3(a). Despite its appearance this is not merely a gravitational well. It represents a spiral well for all material points. At the top of the well $(1 / p \rightarrow 0)$ the interaction potential is dominated by gravity (Fig. 2). At the other extreme $1 / p \rightarrow 1 / 2$ represents very strong attraction dominated by $n \rightarrow \infty$ (usually at small scale, the bottom of the well). In between the material points are compelled to move in the crevices (shown in the inset: Fig. 3(b)). As $n$ increases a body $\varepsilon$ moves further down the well along the spiral. The size of the domain $\ell$ also decreases (i.e. increasing values of $1 / p$ ). The converse is true for larger values of $p$ interacting with lower combined indices $n$ (moving up the spiral). At any location along the spiral the curvature $\nabla s=\nabla \varphi / \varphi$ defines the shape of the crevice (Fig. 3(b)). This consists of a positive slope (its side-wall) and a negative slope (its ridge). The ridge represents the attractive negative potential, whereas the wall corresponds to the repulsive action. Therefore, in a system of size $\ell$, as the curvature of the spiral $\nabla s$ alters, a body $\varepsilon$ undergoes changing acceleration $\Delta a$. The resulting change in inertia would lead to radiation emittance (in accord with Einstein's declaration), hence pulsations $\Delta \varepsilon$. When the aforementioned requisite ratio $p=2 n-1 / n-1$ (for any combination of values of $n$ ) is exceeded the body climbs onto the wall of the crevice. Thus, the path of material points is along the spiral (globally) and to and fro along the slopes of the crevice. This is accompanied by body pulsation and secondary motions, which would determine its inertial properties according to the localized nature of the field. For large bodies such as the earth, the secondary motions can be rotations about their axes, tilting and top-hat spins. Smaller material points are subject to higher interaction potential indices, undergoing wavelike motions with many body spins, as suggested for electrons. At the bottom of the well material points are of insignificant size (almost converted to pure energy) and are subjected to very large attractive potential (elementary particles at fast speed). This inward bound radiation is referred to as anti-Hawking radiation at a radius to the centre of the spiral termed the event horizon. At this location some of the particles will escape (repulsed) and appear as radiation vertically outwards (along the axis of the well). This explanation, known as Hawking radiation, was put forward by Hawking in 1975 [24]. The unified attractive geometric potential can be considered as a general form of gravitation. Consequently, it suggests that material points near strong gravitational potentials are small elementary particles moving at high speeds. When $n \rightarrow \infty$ all bodies with $1 / p \rightarrow 1 / 2$ will be repulsed at $I^{1} \rightarrow 1$ parallel to the axis of the spiral $\left(I^{2}=0\right)$. This agrees with Hawking radiation and European Space Agency's Integral gamma ray Observatory observations of Sagittarius A* [25]. Some other particles continue beyond the event horizon (here set at the limit $1 / p=1 / 2$ ) because of the residual attractive potentials for indices $n<\infty$ (as shown in Fig. 2 for cases $n^{\prime}=3$ and $n^{\prime}=6$ ). These are in line with the supposition of anti-Hawking radiation.

Now returning to the Coulombic potential as an example $K$ assumes the units of $(\mathrm{kg} / \mathrm{C})\left(\mathrm{m}^{3} / \mathrm{s}^{2}\right)$, which differs from the units in the Newtonian gravitational potential $\mathrm{m}^{3} / \mathrm{s}^{2}$. This implies an inherent ratio of mass to charge for material points manifested by permittivity of the medium, which is somehow dependent on body size and distance (i.e. the ratio $\varepsilon / \ell$ ). Using the generic potential described here a Newtonian-type potential can be upheld in which case $1 / \varepsilon_{0}$ would attain the same units as the universal gravitational
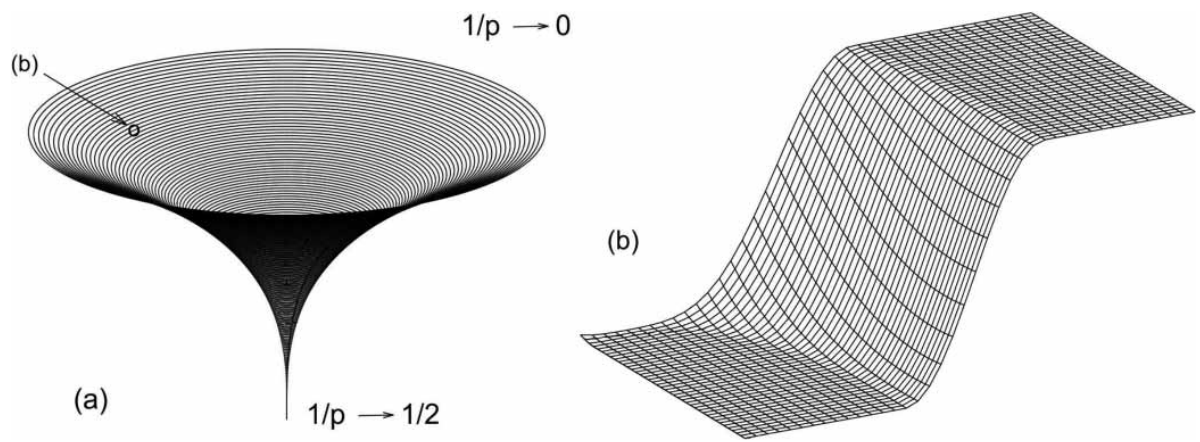

Fig. 3 Implications for motion with the generic unified potential 
constant, but not the same value. This suggests that electrostatics would be operating as a combination of attractive and repulsive interactions with a series of power indices $n$ like the generic potential explained here. The problem with Coulomb's definition is that it implies an elliptic orbit (varying acceleration), which, for example, would mean that negatively charged electrons would have to emit energy and thus finally fall onto the nucleus. However, a combined potential of the form described here removes this anomalous conclusion: with the ascending powers of interaction potential a variable acceleration is achieved. Thus, electrons would have to undergo wavy orbital motions. If the size of the system $\ell$ is fixed the variation in the ratio $1 / p$ in Fig. 2 would be the result of changes in the body size, $\Delta \varepsilon^{n-1}$. The implication is that all bodies would vibrate (quiver/pulsate or undergo secondary motions, depending on their size) in order to remain within the preordained curvatures $\nabla s$.

\section{CONCLUSIONS}

In conclusion, the article has described a generic potential that unifies the weak gravitational force with those of stronger electromagnetic nature at close range. The described potential complies with Newtonian gravitational potential at large distances and for bodies of significant size. In a closed system, it conforms to Mach's principle and arrives at a curvature metric $\nabla s$, which defines the state of motion of all material points within a defined system $\ell$. It further shows that all bodies should vibrate in accelerative and decelerative motions determined by the curvature $\nabla s$ and kinematic conditions given by $I^{1}$, where they are compelled to reside in accordance to their size and most significantly in an implied deterministic manner.

\section{REFERENCES}

1 Galilei, G. Discourses concerning two new sciences, 1638 (Van der Aa, Leiden).

2 Kepler, J. Astronomia nova, 1609 (Prague).

3 Descartes, R. Principia philosophie, 1644 (Elsevier, Amsterdam)

4 Rahnejat, H. Foreword - a tribute to Jean D'Alembert and Albert Einstein: geometrical interpretation of motion: an ironic legacy of apparently irreconcilable atomistic and continuum philosophies. In Multi-body dynamics: monitoring and simulation techniques-III (Eds H. Rahnejat and S. Rothberg), 2004 (Professional Engineering Publishing, London)

5 Newton, I. Philosophiae naturalis principia mathematica, 1687 (Royal Society, London).

6 Leibniz G. Hypothesis physica nova, 1671 (Hanover).

7 Le, J. and D'Alembert, R. Traité de dynamique, 1743 (L’Académie Royal des Sciences, Paris).
8 Euler, L. Nova methodi motum corporum rigidarum determinandi. Novi Commentari Academiae Scientiarum Petropolitanae, 1776, 20, 208-238.

9 Lagrange, J. L. Mécanique analyrique, 1788 (L' Académie Royal des Sciences, Paris).

10 de Coulomb, C. A. Recherches theorique et experimentales sur la force de torsim et l'élasticité des fils de metal, 1784 (L'Académie Royal des Sciences, Paris).

11 Maxwell, J. C. A dynamical theory of the electromagnetic field, 1865 (Royal Society, London).

12 Chandrasekhar, S. Newton's principia, 1995 (Clarendon Press, Oxford).

13 Mach, E. Die mechanik in ihrer entwicklung: Histerischkritisch dagerstellt, 1883 (Leipzig, Brokhaus).

14 Einstein, A. Über den Einfluss der Schwerkraft auf die Ausbreitung des Lichtes. Ann. Phys., 1911, 340(10), 898908.

15 Einstein, A. Zur Elektrodynamik bewegter Körper. Ann. Phys., 1905, 322(10), 891-921.

16 Minkowski, H. Space and time. In The principle of relativity, 1952, pp. 75-91 (Dover Publication, New York).

17 Einstein, A. Ist die Trägheit eines Körpers von seinem Energiegehalt abhängig? Ann. Phys., 1905, 18, 639.

18 Sachs, M. Motion in Einstein's theory of relativity and comparison with classical Views. Proc. IMechE, Part K: J. Multi-body Dynamics, 2005, 219(K2), 125-131.

19 Rahnejat, H. Special relativity: interpretation and implications for space-time geometry. Proc. IMechE, Part K: J. Multi-body Dynamics, 2005, 219(K2), 133-146.

20 Einstein, A. The fundaments of theoretical physics, 1940 (Science, Washington, DC).

21 Einstein, A. Die Grundlage der allgemeinen Relativitätstheorie. Ann. Phys., 1916, 354(7), 769-822.

22 Einstein, A. Newtons Mechanik und ihr Einfluß auf die Gestaltung der theoretischen Physik. Die Naturwissenschafen, 1927, 15(12), 273-276.

23 Bernoulli, J. Opera Johannis Bernoulli, 1742 (Basle).

24 Hawking, S. W. Particle creation by black holes. Commun. Math. Phys., 1975, 43, 199-220.

25 Revnivtsev, M. G., Churazov, E. M., Sazonov, S. Y., Sunyaev, R. A., Lutovinov, A. A., Gilfanov, M. R., Vikhlinin, A. A., Shtykovsky, P. E., and Pavlinsky, M. N. Hard X-ray view of the past activity of Sgr A* in a natural Compton mirror. Eur. J. Astron. Astrophys., 2004, 425, L49-L52.

\section{APPENDIX}

\section{Notation}

a acceleration (scalar)

$\boldsymbol{a}^{i} \quad$ vector of acceleration

$c \quad$ speed of light

C GM

$e_{\mathrm{r}} \quad$ relative permittivity

$e_{0} \quad$ vacuum permittivity

$F \quad$ force

g gravitational acceleration

$\mathbf{g}_{i j} \quad$ co-variant metric tensor (first

fundamental form) 


\begin{tabular}{|c|c|c|c|}
\hline $\mathbf{g}^{k n}$ & contravariant metric tensor & $s$ & arc of motion \\
\hline G & universal gravitational constant & $t$ & time \\
\hline$I^{1}$ & first incompleteness (inverse of Lorentzian & $T$ & kinetic energy \\
\hline & boost) & $\boldsymbol{u}^{i}$ & proper velocity vector on the tangent plane \\
\hline$I^{2}$ & second incompleteness (curvature & $v$ & velocity (scalar) \\
\hline & deviation from the slope of unity) & $\boldsymbol{v}^{i}$ & observed velocity vector on the tangent \\
\hline$\ell$ & size of system/field & & plane \\
\hline$m$ & mass & W & work done \\
\hline$m_{g}$ & gravitational mass & & \\
\hline$m_{I}$ & inertial mass & $\Gamma_{i j, n}$ & Christoffel tensor of the first kind \\
\hline$M$ & mass of a source & $\Gamma_{i j}^{n}$ & Christoffel tensor of the second kind \\
\hline$n$ & $\begin{array}{l}\text { power indices of combinatorial potential } \\
\text { field }\end{array}$ & $\varepsilon$ & size of body (material point) \\
\hline$p$ & system-to-body size ratio & $5<5$ & stationery system) \\
\hline$q$ & charge & $\mu$ & rest mass \\
\hline$q^{i}$ & coordinates on the tangent plane & $\tau$ & local time \\
\hline & (generalized) & $v$ & $v / c$ \\
\hline$r$ & distance from a body $\varepsilon$ & $\varphi$ & potential \\
\hline
\end{tabular}

